(C) 2009 IEEE. Personal use of this material is permitted. Permission from IEEE must be obtained for all other uses, in any current or future media, including reprinting/republishing this material for advertising or promotional purposes, creating new collective works, for resale or redistribution to servers or lists, or reuse of any copyrighted component of this work in other works. 


\title{
Multiuser Subcarrier and Power Allocation Algorithm for OFDM/Offset-QAM
}

\author{
K. El Baamrani, V. P. Gil Jiménez, Member, IEEE, A. G. Armada, Senior Member, IEEE, and A. A. Ouahman
}

\begin{abstract}
In this letter, the multiuser bit and power allocation problem for an Orthogonal Frequency Division Multiplexing (OFDM)/Offset-Quadrature Amplitude Modulation (OQAM) system is investigated. The Signal-to-Interference-plus-Noise Ratio (SINR) and required power expressions for the formulation of the problem in an OFDM/OQAM system are developed. Next, a suboptimal algorithm based on this formulation is proposed.
\end{abstract}

Index Terms-OFDM/OQAM, power allocation, SNIR.

\section{INTRODUCTION}

$\mathbf{T}$ HERE ARE two modulations mainly used in OFDM systems, namely, Quadrature Amplitude Modulation (QAM) and Offset-QAM (OQAM) [1]-[5]. On one side, when employing QAM modulation with a rectangular pulse shape of the OFDM symbol, robustness against multipath is achieved by using Cyclic Prefi (CP). This classic scheme will be referred to as OFDM/QAM. On the other side, OFDM with well-localized pulse shapes in time and frequency has been proposed [4], [5]. This scheme will be referred to as OFDM/OQAM. As is the case for OFDM/QAM, efficien implementation based on FFT is also possible [4], [5]. OFDM/OQAM is a filtere modulation where in-phase and quadrature components are delayed by half a symbol period. It does not require guard interval and thus, a higher spectral efficien y and a throughput increase are expected. The fact that OFDM/OQAM outperforms OFDM/QAM has been pointed out in [1], [2], [6], [7].

In single user OFDM systems, several algorithms for adaptive bit and power allocation have been developed, e.g., [8], [9]. However, from the knowledge of the authors, there are no algorithms for bit and power allocation for OFDM/OQAM. In these systems, Inter-Carrier Interference (ICI) and Inter-Symbol Interference (ISI) will appear due to the absence of CP, and thus, the classical formulations that relate the error probability, number of bits/symbol and required power for OFDM/QAM cannot be directly applied anymore. In this letter we firs develop a new formulation for the Signal-to-Noise-plus-Interference Ratio (SNIR) and, based on it, we obtain a relationship between the required power and the number of bits/symbol. Next,

work supported in part by Project TEC2008-06327-C03-02 and AECI.

This

K. El Baamrani and A. A. Ouahman are with the Department of telecommunications, ENSA. University of Cadi Ayyad, Marrakech, Morocco (e-mail: elbaamrani@ucam.ac.ma; ouahman@ucam.ac.ma).

V. P. G. Jiménez and A. G. Armada are with the Department of Signal Theory and Communications, University Carlos III of Madrid, Madrid, Spain (e-mail: vgil@tsc.uc3m.es; agarcia@tsc.uc3m.es). we develop a new algorithm for OFDM/OQAM and we show that it outperforms OFDM/QAM with [10]-[13] and OFDM/ OQAM with [10].

Notations: Matrices and vectors are represented by bold-face capitals and bold-face letters respectively, $\left(^{\sim}\right)$ denotes paraconjugation, viz. $\tilde{G}(z)=G^{*}\left(z^{-1}\right)$, estimations are represented by $\left({ }^{\top}\right)$, superscripts ()$^{T},()^{*},()^{R}$, and ()$^{I}$ denote transpose, conjugation, real and imaginary part respectively, $\lfloor$.$\rfloor means the in-$ teger part whereas $\sim$ means proportional.

\section{SYSTEM DESCRIPTION}

We consider an OFDM/OQAM system with $K$ users where the total available bandwidth is divided into $N$ orthogonal subcarriers and each transmitter can use the number of subcarriers that are required to satisfy its own bit rate. Each subcarrier conveys a series of complex QAM symbols of $T$ seconds duration. The transmitted $n$th complex symbol in subcarrier $m$ for user $k$ is $c_{k, m, n}=c_{k, m, n}^{R}+j c_{k, m, n}^{I}$. Offset QAM is attained by delaying the imaginary and real part of each symbol by $T / 2$ in even and odd subcarriers respectively. Pulse shaping of the transmitted signal is achieved by a transmitter filte $\xi(t)$. The transmitted signal $s(t)$ can be written as [4]

$$
\begin{aligned}
s(t)= & \sum_{n} \sum_{k=1}^{K} \sum_{m=0}^{\frac{N}{2}-1}\left\{\left[c_{k, 2 m, n}^{R} \xi(t-n T)\right.\right. \\
& \left.+j c_{k, 2 m, n}^{I} \xi(t-T / 2-n T)\right] e^{j 2 \pi(2 m) F t} \\
& +\left[j c_{k, 2 m+1, n}^{I} \xi(t-n T)\right. \\
& \left.\left.+c_{k, 2 m+1, n}^{R} \xi(t-T / 2-n T)\right] e^{j 2 \pi(2 m+1) F t}\right\}
\end{aligned}
$$

where $F=1 / T$ is the spacing between two subcarriers.

Adopting the notations $a_{k, 2 m, 2 n}=c_{k, 2 m, n}^{R}, a_{k, 2 m, 2 n+1}=$ $c_{k, 2 m, n}^{I}, a_{k, 2 m+1,2 n}=c_{k, 2 m+1, n}^{I}$ and $a_{k, 2 m+1,2 n+1}=$ $c_{k, 2 m+1, n}^{R}$, we can rewrite (1) in discrete time as [5]

$$
s[p]=\sum_{n} \sum_{k=1}^{K} \sum_{m=0}^{N-1} a_{k, m, n} \gamma_{m, n}[p]
$$

where $a_{k, m, n}$ is the $n$th real symbol transmitted in subcarrier $m$ for user $k, p$ is $\gamma_{m, n}[p]=$ $\xi[p \quad-\quad n M] e^{j \varphi_{m, n}} e^{j(2 \pi / 2 M) m(p-(L-1) /(2))}$ being $\varphi_{m, n}=(m+n) \pi / 2$ and $M$ is half the number of subcarriers. The received signal for $k$ th user is

$$
r_{k}[p]=s[p] * h_{k}[p]+n_{0}[p]
$$

where $h_{k}[p]$ is the channel response for user $k$ assumed constant within the OFDM symbols that conform a packet (block fading), and $n_{0}[p]$ is the Additive White Gaussian Noise (AWGN). After FFT, the received signal for user $k$ can be expressed as 


$$
r_{k}[p]=\sum_{n} \sum_{m=0}^{N-1} a_{k, m, n} H_{k, m} \gamma_{m, n}[p]+n_{0}[p]
$$

where $H_{k, m}$ is the coefficien of the channel frequency response for user $k$ in subcarrier $m$.

\section{SINR AND POWER EXPRESSIONS}

In this section, the SINR for the OFDM/OQAM system is derived and the required power to convey a certain number of bits/ symbol is developed. Let us denote by $x_{k, m, n}=a_{k, m, n} H_{k, m}$ and $\hat{x}_{k, m, n}=\hat{a}_{k, m, n}$. Let us assume that the number of subcarriers $N$ is even $(N=2 M)$. From (42) in [5], the received symbols can be expressed as

$\hat{x}_{k, m, n}=\overbrace{q_{0}(0) a_{k, m, n} H_{k, m}}^{\text {desired signal }}+\left.\breve{x}_{k, m, n}\right|_{\text {ISI }}+\left.\breve{x}_{k, m, n}\right|_{\text {ICI }}+n_{0}$

where

$$
\begin{aligned}
\left.\breve{x}_{k, m, n}\right|_{\mathrm{ISI}}= & \sum_{j \neq 0} q_{0}(j) a_{k, m, n-j} H_{k, m}, \\
\left.\breve{x}_{k, m, n}\right|_{\mathrm{ICI}}= & \sum_{j} \sum_{\ell=1}^{\lfloor m / 2\rfloor} q_{\ell}(j) a_{k, m-2 \ell, n-j} H_{k, m-2 \ell} \\
& +\sum_{j} \sum_{\ell=1}^{M-\lfloor m / 2\rfloor-1} q_{\ell}(j) a_{k, m+2 \ell, n-j} H_{k, m+2 \ell}
\end{aligned}
$$

and $q_{\ell}$ is the inverse z-transform of $Q_{\ell}$ with

$$
\begin{aligned}
Q_{\ell}(z)= & 2(-1)^{\ell} \sum_{o=0}^{M-1}\left(G_{o}\left(-z^{2}\right) \tilde{G}_{o}\left(-z^{2}\right)+\right. \\
& \left.+G_{\bar{o}}\left(-z^{2}\right) \times \tilde{G}_{\bar{o}}\left(-z^{2}\right)\right) \cos \left[\frac{\pi}{M} \ell(2 o-L+1)\right]
\end{aligned}
$$

$\bar{o}=M+o$ and $G_{\ell}(z)=\sum_{n} \xi[\ell+2 n M] z^{-n}$. Assuming without loss of generality, that the data symbols are statistically independent between different subcarriers, different symbols, and between real and imaginary parts. Then we can obtain expressions for the instantaneous power of ISI and ICI as follows.

Let $P_{k, m}(n)$ the transmission power for user $k$ on subcarrier $m$ at $n$th symbol. By taking $\Xi_{k, m, \ell}(n)=H_{k, m+2 \ell}^{2} P_{k, m+2 \ell}(n)$, we can write the ISI and ICI power as

$$
\begin{aligned}
\left.P_{k, m}\right|_{\text {ISI }}= & \sum_{j \neq 0}\left|q_{0}(j)\right|^{2} \Xi_{k, m, 0}(n-j) \\
\left.P_{k, m}\right|_{\text {ICI }}= & \sum_{j} \sum_{\ell=1}^{\lfloor m / 2\rfloor}\left|q_{\ell}(j)\right|^{2} \Xi_{k, m,-\ell}(n-j) \\
& +\sum_{j} \sum_{\ell=1}^{M-\lfloor m / 2\rfloor-1}\left|q_{\ell}(j)\right|^{2} \Xi_{k, m, \ell}(n-j) .
\end{aligned}
$$

The SINR for user $k$ in subcarrier $m$ can be expressed as

$$
\operatorname{SINR}_{k, m}(n)=\frac{\left|q_{0}(0)\right|^{2} H_{k, m}^{2} P_{k, m}(n)}{\left.P_{k, m}\right|_{\mathrm{ISI}}+\left.P_{k, m}\right|_{\mathrm{ICI}}+N_{0}(n)}
$$

where $N_{0}(n)$ is AWGN noise. The interference terms (6) and (7) include the summation of a large amount of OFDM symbols. Therefore, they approach a Gaussian signal and then, the number of transmittable bits/symbol $b_{k, m}$ may be approximated by

$$
b_{k, m}(n)=\log _{2}\left(1+\frac{\operatorname{SINR}_{k, m}(n)}{\Gamma}\right)
$$

where $\Gamma$ is the signal-to-noise ratio gap that depends on the Symbol Error Rate (SER), the noise margin and the coding gain [12]. For M-QAM modulation it can be approximated ${ }^{1}$ as $\Gamma=(1 / 3)\left[Q^{-1}((\mathrm{SER}) /(4))\right]^{2}$. Alternatively, an expression such as the one developed in [14] can be used.

Now, after the development of the SINR, we can obtain an expression that relates the number of bits/symbol and required power. From (11) and (12) we can write

$P_{k, m}(n)=\frac{\left(2^{b_{k, m}(n)}-1\right) \Gamma}{H_{k, m}^{2}\left|q_{0}(0)\right|^{2}}\left(N_{0}(n)+\left.P_{k, m}\right|_{\mathrm{ISI}}+\left.P_{k, m}\right|_{\mathrm{ICI}}\right)$.

It should be noted that (13) establishes a relationship between all the variables (the powers $P_{k, m}(n)$ which determine the subcarrier assignment, and the number of bits $\left.b_{k, m}\right)$ and the interference. From (9), (10), and (13) we get

$$
\begin{aligned}
P_{k, m}( & n)-\frac{\left(2^{b_{k, m}(n)}-1\right) \Gamma}{H_{k, m}^{2}\left|q_{0}(0)\right|^{2}}\left[\sum_{\ell=1}^{\lfloor m / 2\rfloor}\left|q_{\ell}(0)\right|^{2} \Xi_{k, m,-\ell}(n)\right. \\
& \left.+\sum_{\ell=1}^{M-\lfloor m / 2\rfloor-1}\left|q_{\ell}(0)\right|^{2} \Xi_{k, m, \ell}(n)\right] \\
= & \frac{\left(2^{b_{k, m}(n)}-1\right) \Gamma}{H_{k, m}^{2}\left|q_{0}(0)\right|^{2}}\left[N_{0}(n)+\sum_{j \neq 0}\left|q_{0}(j)\right|^{2} \Xi_{k, m, 0}(n-j)\right. \\
& +\sum_{j \neq 0}^{\lfloor m / 2\rfloor} \sum_{\ell=1}^{\lfloor m}\left|q_{\ell}(j)\right|^{2} \Xi_{k, m,-\ell}(n-j) \\
& \left.+\sum_{j \neq 0}^{M-\lfloor m / 2\rfloor-1} \sum_{\ell=1}^{M}\left|q_{\ell}(j)\right|^{2} \Xi_{k, m, \ell}(n-j)\right] .
\end{aligned}
$$

Through (14), we observe that $P_{k, m}(n)$ depends on $P_{k, m}(n-j)$. Thus, we arrange the power in a compact matrix form

$$
\mathbf{P}(n)=\boldsymbol{\Theta}(n)(\mathbf{A}(n))^{-1}\left(N_{0}+\sum_{j \neq 0} \mathbf{B}(n-j) \mathbf{P}(n-j)\right)
$$

where

$$
\boldsymbol{\Theta}_{k, m}(n)=\frac{\left(2^{b_{k, m}(n)}-1\right) \Gamma}{H_{k, m}^{2}\left|q_{0}(0)\right|^{2}}
$$

and (see (16) and (17) at the bottom of the next page).

\section{Subcarrier, BIT AND POWER Allocation}

Let us defin $S_{k} \subset\{0,1, \cdots, N-1\}$ the set of subcarriers for user $k$. The objective is to fin the best assignment of $b_{k, m}$

\footnotetext{
${ }^{1}$ In this letter we have chosen without loss of generality $\Gamma$ to be constant in time and equal for all the users for the sake of simplicity, but $\Gamma$ may depend on the user and also it may be adapted in time $\left(\Gamma_{k, m}(n)\right) . \infty^{-\left(\left(t^{2}\right) /(2)\right)}$.
} 
TABLE I

Sub-CARrier, Bit AND POWER Allocation Algorithm

\begin{tabular}{|c|c|}
\hline (a) Sub-carriers Allocation Stage & (b) Bit and Power Allocation Stage \\
\hline $1:$ Set $\Omega=0,1, \ldots, N-1$ & $1:$ Set: $\Psi=1,2, \ldots, K$ \\
\hline$P_{k}=0 \quad \forall k$ & $\Omega=0,1, \ldots, N-1$ \\
\hline $\begin{array}{l}\text { 2: Evaluate } \Delta P_{k, m}(0) \text { using (21) } \forall k, m \\
\text { for } k=1 \text { to } K\end{array}$ & $\begin{array}{l}b_{k, m}=0 \forall k, m \\
R_{T}=\sum_{k=1}^{K} R_{k}\end{array}$ \\
\hline 3: $\quad j=\arg \min _{m, \in \Omega} \Delta P_{k, m}(0)$ & while $\sum_{m=0}^{N-1} \sum_{k=1}^{K} b_{k, m}<R_{T}$ \\
\hline 4: $\quad P_{k}=P_{k}+\Delta P_{k, j}(0)$ & 2: $\quad j=\arg \min _{k \in \mathbb{X}} \Delta P_{k, m}$ \\
\hline 5: $\quad \Omega=\Omega-j$ & 3: $\quad k=$ The user to which one \\
\hline $\begin{array}{l}\text { 6: } \quad S_{k}=S_{k}+j \\
\text { end for }\end{array}$ & $\begin{array}{l}\text { allocated the sub-carrier } j \\
\text { if } \sum_{m=0}^{N-1} b_{k, m}<R_{k}\end{array}$ \\
\hline while $\Omega \neq \phi$ & $b_{k, j}=b_{k, j}+1$ \\
\hline 7: $\quad k=\arg$ nin $P_{k}$ & Calculate $A(n)$ using (22) \\
\hline 8: $\quad j=\arg \operatorname{mini1}_{m \in \Omega 2} \Delta P_{k, m}(0)$ & Calculate $P(n)$ using (15) \\
\hline 9: $\quad \Omega=\Omega-j$ & Update $\Delta P_{k, j}\left(b_{k, j}\right)$ using (20) \\
\hline 10: $\quad S_{k}=S_{k}+j$ & else \\
\hline 11: $\quad P_{k}=P_{k}+\Delta P_{k, j}(0)$ & 8: $\quad \Psi=\Psi-k$ \\
\hline end while & $\begin{array}{l}\text { 9: } \quad \Omega=\Omega-S_{k} \\
\text { end if } \\
\text { end while }\end{array}$ \\
\hline 12: OUTPUT $\left\{S_{k}, P_{k}\right\}_{k=1}^{K}$ & 10: OUTPUT $\left\{b_{k, m}\right\}_{k=1, m=1}^{K . N}$ \\
\hline
\end{tabular}

so that the total power is minimized given each user's target bit rate. The problem can be formulated as follows

$$
\begin{aligned}
\text { Minimize } & \sum_{k=1}^{K} \sum_{m=0}^{N-1} P_{k, m} \\
\text { Subject to : } & \sum_{m \in S_{k}} b_{k, m} \geq R_{k} \quad \forall k=1, \ldots, K \\
& P_{k, n} \geq 0 \quad \forall k, n \\
& S_{i} \bigcap S_{j}=\phi \quad \forall i \neq j \\
& S_{1} \bigcup S_{2} \bigcup \cdots \bigcup S_{M} \subset\{0,1, \ldots, N-1\} .
\end{aligned}
$$

Where $R_{k}$ represents the required bit rate by user $k$. $S_{1}, \ldots, S_{k}$ need to be disjoint since each subcarrier can only be occupied by only one user. We present our approach to solve this problem in two steps. First, the subcarriers are allocated to the users and the bits are assigned to subcarriers one bit at a time.

\section{A. Subcarriers Allocation Algorithm}

In firs step, we keep allocating to the user that has the minimum total power an unoccupied subcarrier that requires the least additional power. Let $\Delta P_{k, m}\left(b_{k, m}\right)$ denotes the incremental power, i.e., additional power needed for transmitting one additional bit on subcarrier $m$ for user $k$, when the number of bits currently loaded is $b_{k, m}$. Thus

$$
\Delta P_{k, m}\left(b_{k, m}\right)=P_{k, m}\left(b_{k, m}+1\right)-P_{k, m}\left(b_{k, m}\right)
$$

with, $\Delta P_{k, m}(0)=P_{k, m}\left(b_{k, m}=1\right)$. Then, from (15) and (19), we obtain $\Delta P_{k, m}(0) \sim 1 / H_{k, m}^{2}$. By taking $\Upsilon_{k, m}=$ $H_{k, m}^{2} / \sum_{n=0}^{N-1} H_{k, n}^{2}$, we can write

$$
\Delta P_{k, m}(0) \sim 1 / \Upsilon_{k, m}
$$

\section{B. Bit and Power Allocation Algorithm}

In second step, after all subcarriers have been assigned, we start by looking for the subcarrier that requires the smallest incremental power to accommodate one more bit. We add an additional bit and increase the power on that subcarrier to the user to which it was assigned. Next, the total required power for all users is recalculated according to (19). Then, we iterate this process until all the constraints of the optimization problem are met. In order to update the total required power we need to calculate $\mathbf{P}(n)$ by using (15). However, the calculation of $\mathbf{P}(n)$ requires the inversion of $\mathbf{A}(n)$. The complexity in the calculation of $\mathbf{A}(n)^{-1}$ can be reduced using $\mathbf{A}(n-1)^{-1}$. At $n=0$, we have $\mathbf{A}(0)=\mathbf{I}_{\mathbf{N}}\left(\mathbf{I}_{\mathbf{N}}\right.$ is the $N \times N$ identity matrix $)$ then, $A(n)^{-1}$ can be easily updated as

$$
\begin{aligned}
(\mathbf{A}(n))^{-1}=\mathbf{A}(n-1)^{-1} & \\
& -\frac{\left(\mathbf{A}(n-1)^{-1} \mathbf{v}\right)\left(\mathbf{z}^{T} \mathbf{A}(n-1)^{-1}\right)}{1+\mathbf{z}^{T} \mathbf{A}(n-1)^{-1} \mathbf{v}}
\end{aligned}
$$

where $\mathbf{v}$ is a vector whose $m$ th element is $\boldsymbol{\Theta}_{k, m}$ and all other are 0 , and $\mathbf{z}$ is the $m$ th column of the following matrix $\mathbf{Z}$

$$
Z_{i, j}(n-k)=\left\{\begin{array}{ll}
0, & \text { if } i=j \text { or } i-j \text { odd } \\
\left|q_{\frac{i-j}{2}}(k)\right|^{2} H_{i, j}^{2}, & \text { if } i-j \text { even and } j \leq i \\
\left|q_{\frac{j-i}{2}}(0)\right|^{2} H_{i, j}^{2}, & \text { if } i-j \text { even and } j \geq i
\end{array} .\right.
$$

The algorithm is described by the pseudocode in Table I

\section{Complexity}

The complexity of the proposed algorithm is $\mathcal{O}(K N)+$ $\mathcal{O}(2 N)$. These values are of the same order as in [9]-[12] and [13]. The complexities in [9], [10] and [11] are $\mathcal{O}(2 K N), \mathcal{O}\left(I_{1} K N+I_{2} K\right)$, and $\mathcal{O}(2 K N+N \log N)$ respectively, where $I_{1}$ and $I_{2}$ are the number of iterations in the inner and outer loop that they use. The complexities in [12] and [13] are $\mathcal{O}(K N)+\mathcal{O}(N)$ and $\mathcal{O}\left(I K N+N^{2}\right)$ respectively, where $I$ is the number of iterations.

\section{PERformance AnAlysis}

In this section, the OFDM/QAM system uses $N=512$ subcarriers and a cyclic prefi of $1 / 8$ of the OFDM symbol duration, whereas the OFDM/OQAM system uses the same number of subcarriers but no cyclic prefix and the family of pulses introduced by Vahlin and Holte in [15]. The target SER was fi ed

$$
\begin{array}{r}
A_{k, m}(n)= \begin{cases}1, & \text { if } k=m \\
-\Theta_{k, m}(n)\left|q_{\frac{k-m}{2}}(0)\right|^{2} H_{k, m}^{2}, & \text { if } k-m \text { even and } m \leq k \\
-\Theta_{k, m}(n)\left|q_{\frac{m-k}{2}}(0)\right|^{2} H_{k, m}^{2}, & \text { if } k-m \text { even and } m \geq k \\
0, & \text { if } k-m \text { odd }\end{cases} \\
B_{k, m}(n-j)= \begin{cases}\left|q_{0}(j)\right|^{2} H_{k, m}^{2}, & \text { if } k=m \\
\left|q_{\frac{k-m}{2}}(j)\right|^{2} H_{k, m}^{2}, & \text { if } k-m \text { even and } m \leq k \\
\left|q_{\frac{m-k}{2}}(0)\right|^{2} H_{k, m}^{2}, & \text { if } k-m \text { even and } m \geq k \\
0, & \text { if } k-m \text { odd. }\end{cases}
\end{array}
$$




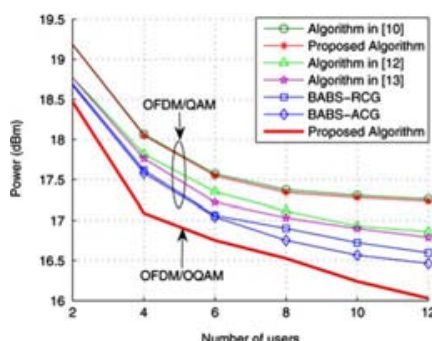

(a)

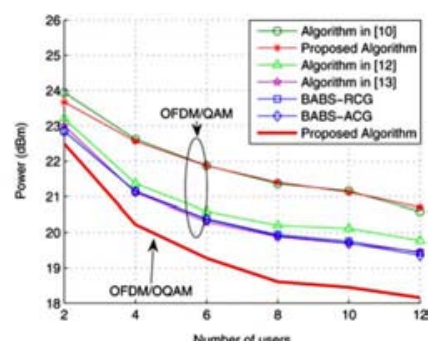

(b)
Fig. 1. Total transmit power for scenario A: (a) PA and (b) VA.

to $10^{-4}$ and the power of AWGN was $-50 \mathrm{dBw} / \mathrm{Hz}$. Two scenarios have been evaluated. In scenario $\mathrm{A}$, the total rate $R_{T}$ was fi ed to 2048 bits/OFDM symbol and $R_{k}=\left(R_{T} / K\right)$ for all $k$. And, in scenario $\mathrm{B}$, the user data rate has been fi ed to $R_{k}=200$ bits/OFDM symbol, and thus $R_{T}=K \times R_{k}$. The overall bandwidth for both scenarios was $7.68 \mathrm{MHz}$. Two ITU channel models have been used for simulations, Pedestrian A (PA) at $3 \mathrm{~km} / \mathrm{h}$ and Vehicular A (VA) at $50 \mathrm{~km} / \mathrm{h}$.

In Fig. 1, the total transmit power required to achieve the target bit rate in scenario $\mathrm{A}$ is shown. Due to the decrease in user data rate and also to the multiuser diversity, the total transmit power decreases as the number of users is increased, especially in the proposed algorithm for OQAM. This effect can be better observed in scenario B [Fig. 2(a)], where users require a constant data rate independent of the number of users) and the slope of the proposed algorithm is smaller than linear. Also the performance of the proposed algorithm applied to classical OFDM/QAM is shown in these figures It can be seen that the proposed algorithm outperforms the others, especially as the number of users increases, mainly due to the higher efficien $y$ of OFDM/OQAM; However, the algorithm here developed also outperforms others previously available for the same modulation. To illustrate this fact, in Fig. 2(b), a comparison of applying an existing algorithm [10] for Pedestrian A, scenario A with our developed SINR expressions in Section III to OQAM, and our proposal is shown. The algorithm has a reduced complexity but the results in terms of power efficien y are worse than [11]-[13]. Unfortunately, we cannot combine [11]-[13] with our expression of Section III since they assume independence between the powers assigned to subcarriers. It can be observed in Fig. 2(b) that the proposed algorithm performs better than [10] with OFDM/OQAM. On the other hand, we can see in Fig. 1 that, when it is applied to classical OFDM/QAM, our algorithm performs similar to [10]. Consequently, we can conclude that the proposed algorithm is a successful solution to the problem of power minimization for an OFDM/OQAM system.

\section{CONCLUSION}

In this letter, the SINR and required power expressions have been developed and a multiuser bit and power allocation algorithm for OFDM/OQAM downlink transmission has been presented. Simulation results show that the proposed algorithm offers better performance than previous OFDM/QAM schemes allowing to overcome some of the efficien $y$ problems that

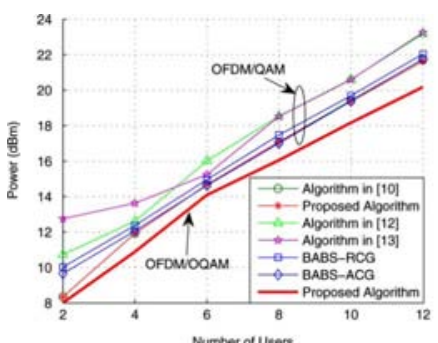

(a)

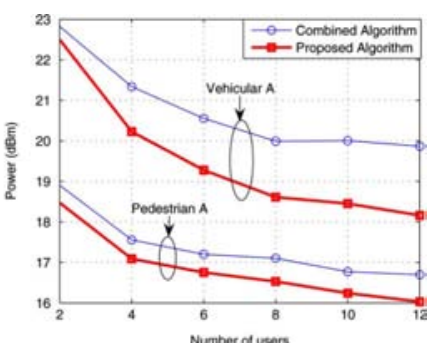

(b)
Fig. 2. Total transmit power: (a) Scenario B (VA) and (b) Combined (PA).

OFDM/QAM suffers from. Besides, our proposal is shown to be more energy-efficien than a previous algorithm when applied to OFDM/OQAM and the complexity is similar to previous algorithms in the literature.

\section{REFERENCES}

[1] H. Zhang, D. Le Ruyet, and M. Terre, "Spectral efficien y analysis in OFDM and OFDM/OQAM based cognitive radio networks," in Proc. IEEE Vehicular Tech. Conf. (VTC), Apr. 26-29, 2009, vol. 1.

[2] A. B. Salem, M. Siala, and H. Boujemaa, "Performance comparison of OFDM and OFDM/OQAM systems operating in highly time and frequency dispersive radio-mobile channels," in Proc. of IEEE Int. Conf. on Elect., Systems and Circuits (ICECS), Dec. 11-14, 2005.

[3] T. Fusco, L. Izzo, A. Petrella, and M. Tanda, "Blind symbol timing estimation for OFDM/OQAM systems," IEEE Trans. Signal Process., 2009, In press.

[4] B. Hirosaki, S. Hasegawa, and A. Sabato, "Advanced groupband data modem using orthogonally multiplexed QAM technique," IEEE Trans. Commun., vol. 34, no. 6, pp. 587-592, June 1986.

[5] P. Siohan, C. Siclet, and N. Lacaille, "Analysis and design of OFDM/ OQAM systems based on filterban theory," IEEE Trans. on Signal Proc., vol. 50, no. 5, pp. 1170-1183, May 2002.

[6] J. Du and S. Signell, "Comparison of CP-OFDM and OFDM/OQAM in doubly dispersive channels," in Proc. IEEE Future Generation Comm. and Networking (FGCN), Dec. 2007.

[7] D. Lacroix, N. Goudard, and M. Alard, "OFDM with guard interval versus OFDM/OffsetQAM for high data rate UMTS downlink transmission," in Proc. IEEE Vehicular Tech. Conf., Oct. 2001, vol. 4, no. 7-11, pp. 2682-2686.

[8] D. H. Hartogs, "Ensemble Modem Structure for Imperfect Transmission Media," U.S. patents 4679227 July 1987, 4731816 Mar. 1988, 4833706 May 1989.

[9] C. Y. Wong, R. S. Cheng, K. B. Letaief, and R. D. Murch, "Multiuser OFDM with adaptive subchannel, bit, and power allocation," IEEE J. Sel. Areas Commun., vol. 17, no. 10, pp. 1747-1758, Oct. 1999.

[10] I. Kim, H. L. Lee, B. Kim, and Y. H. Lee, "On the use of linear programming for dynamic subchannel and bit allocation in multiuser OFDM," in Proc. IEEE Global Conf. Commun. (GLOBECOM), Nov. 2001, vol. 6, pp. 3648-3652.

[11] D. Kivanc, L. Guoqing, and H. Liu, "Computationally efficien bandwidth allocation and power control for OFDMA," IEEE Trans. Wireless Commun., vol. 2, no. 6, pp. 1150-1158, Nov. 2003.

[12] K. El Baamrani, V. P. G. Jiménez, A. G. Armada, A. A. Ouahman, and S. Allaki, "Subcarrier and power allocation for the downlink of multiuser OFDM transmission," Wireless Pers. Commun., vol. 39, no. 4 , pp. 457-465, Dec. 2006, Springer.

[13] Z. YuMing, G. JingBo, W. ZanJi, and X. Yi, "A novel sub-carrier and power allocation algorithm for multi-user OFDM systems," in Proc. IEEE Int. Conf. on Commun. (ICC), June 2006, vol. 12, pp. 5639-5642.

[14] A. J. Goldsmith and S. G. Chua, "Variable-rate variable-power MQAM for fading channels," IEEE Trans. Commun., vol. 45, no. 10, pp. 1218-1230, Oct. 1997.

[15] A. Vahlin and N. Holte, "Optimal finit duration pulses for OFDM," IEEE Trans. Commun., vol. 44, no. 1, pp. 10-14, Jan. 1996. 